\title{
Structural correlations in disordered matter: An experimental separation of orientational and positional contributions
}

\author{
F. J. Bermejo, A. Criado, R. Fayos, and R. Fernández-Perea \\ Consejo Superior de Investigaciones Cientificas, Serrano 123, Madrid E-28006, Spain \\ H. E. Fischer and E. Suard \\ Institut Laue Langevin, Boîte Postal 156x, F-38042 Grenoble Cedex 9, France \\ A. Guelylah and J. Zúñiga \\ Departamento de Física de la Materia Condensada, Universidad del Pais Vasco, Facultad de Ciencias, E-48960 Bilbao, Spain
}

(Received 27 February 1997; revised manuscript received 20 May 1997)

\begin{abstract}
The structures of the liquid, glass (i.e., amorphous), rotator-phase (RP) crystal, orientational glass (OG), and the stable (monoclinic) crystal phases of ethanol are investigated by means of neutron and x-ray diffraction. The RP crystal and OG phases show an intermediate kind of disorder between that of the fully disordered glass and liquid phases and that of the stable crystal. The Bragg pattern of the RP and OG crystals provides information about the time-averaged distribution of single-molecule orientations, and a contribution comprising intermolecular correlations is isolated from the diffuse intensity. Finally, the $\mathrm{RP} \rightarrow \mathrm{OG}$ rotational freezing transition is analyzed in detail and shown to constitute a physical realization of the glass transition found in hard-needle models. [S0163-1829(97)05441-6]
\end{abstract}

\section{INTRODUCTION}

The purpose of the present work is to provide quantitative details on the static correlations of ethyl alcohol, a material which can be prepared under the very same thermodynamic conditions in phases showing topological (liquid and amorphous) or purely orientational disorder (rotator phase and orientational glass). ${ }^{1-3}$ The relevance of such a study stems from the fact that knowledge of the structural arrangements in phases showing such rather different types of disorder may be of help to understand the role played by orientational and positional correlations, at temperatures where structurally arrested phases (glass and orientational glass) exist as well as those about the liquid $\rightarrow$ glass and rotator $\rightarrow$ orientational-glass transitions. Consequently, studies on this material can help to bridge the gap between those carried out for fully disordered phases (conventional glasses and liquids in either normal or supercooled states) and those regarding orientationally disordered crystals. It can therefore be expected that such studies may shed some light on the distinctive roles played by the loss of orientational and positional correlations as relevant sources of disorder needed to account for the most characteristic properties of the amorphous state.

As known since nearly two decades ago, ${ }^{2}$ ethanol exhibits an interesting polymorphism leading to phases which can be produced depending upon temperature and cooling rates. The liquid after supercooling forms a topologically disordered solid (glass) if the temperature is decreased below about $T_{g}=97 \mathrm{~K}$ or ends in a "glassy crystal" phase if it is left to anneal at temperatures $97 \mathrm{~K} \geqslant T \geqslant 115 \mathrm{~K}$. Such a crystalline phase $^{2}$ then undergoes an additional "glass transition'" also at $\approx 97 \mathrm{~K}$, below which an "orientational-glass" phase appears which shows no further transformation upon subsequent cooling down to the lowest explored temperatures $(1.5$
K). Alternatively, the stable, monoclinic orientationally ordered phase is easily prepared by annealing the liquid within the $120-158 \mathrm{~K}$ range.

Our interest here is also motivated by the possibility of quantifying the extents of orientational and positional regularities, something which may have consequences relevant to ordering in glasses at intermediate ranges (IRO) (e.g., above that characterized by direct bonding). In fact, as shown in a preliminary communication, ${ }^{4}$ knowledge of the ordering characteristics in the RP and OG phases provides valuable keys for the understanding of that present in the glass and liquid phases, a topic discussed in extenso for several decades $^{5}$ now. Moreover, the similarities between disordered crystal and amorphous phases are not confined to structural aspects since striking similarities between some behaviors of the RP and OG phases and those of the topologically disordered solid are also evident in some macroscopic properties such as the specific heat and thermal conductivity. ${ }^{3}$

Among the previous results on the structure of the different solid phases, the paper of Jönsson ${ }^{6}$ on the structure of the stable (monoclinic) crystal as studied by x-ray diffraction of single crystals constitutes the most solid piece of experimental work. Some investigations regarding the microscopic structure of the liquid have also appeared, ${ }^{7}$ although the obtained results concerning the short-range intermolecular ordering are somewhat dependent on the way the singlemolecule contribution $f_{1}(Q)$ is subtracted from the total $S(Q)$ structure factor, a step which needs to be taken since the characteristic distances of closest approach between nearest neighbors turn out to be shorter than some of those separating nuclei belonging to the same molecule. The structure of the RP and OG phases was recently established as corresponding to bcc lattices ${ }^{1}$ on the basis of $x$-ray ${ }^{1}$ and neutron ${ }^{4}$ measurements. Only two reflections located at $1.65 \AA^{-1}$ (very intense) and $2.33 \AA^{-1}$ (weak) were visible as very 
sharp Bragg peaks in the $\mathrm{x}$-ray measurements ${ }^{1}$ whereas up to six reflections could be disentangled from the strong diffuse scattering in the neutron experiments. ${ }^{4}$ Above $\approx 97 \mathrm{~K}$ the crystal belongs to the "plastic" or rotator-phase (RP) compounds, as evidenced by a strong, broad quasielastic signal appearing in the inelastic neutron-scattering spectra. ${ }^{8}$ With two molecules per unit cell and a lattice constant of $5.3 \AA$, the RP phase shows a macroscopic mass density of about $\rho_{m}=958.2 \mathrm{Kg} \mathrm{m}^{-3}$ (depending on temperature), far closer to that of the glass or supercooled liquid than to the stable crystal $\left(\rho_{m}=1025 \mathrm{Kg} \mathrm{m}^{-3}\right.$ at $\left.87 \mathrm{~K}\right)$. A calorimetric transition $^{2}$ separates the RP from a phase with a remarkably similar structure ${ }^{4}$ where the rotational background is absent, at least on a meV scale, and therefore seems to represent a frozen, or orientational-glass $(\mathrm{OG})$ state.

Clarifying the nature of the $\mathrm{RP} \rightarrow \mathrm{OG}$ freezing transition is also one of the objectives of the present communication. The available data (mainly from differential scanning calorimetry, ${ }^{2}$ broad band $\mathrm{NMR}^{8}$ Raman and $\mathrm{x}$-ray diffraction ${ }^{1}$ ) do not show any stark difference in any property examined at both sides of the transition, account made of thermal effects. From such a basis, the transition is known to take place within a range of temperatures 90-98 K, remarkably close to that where the $T_{g}$ signalling the liquid-glass transition occurs. To shed more light on this point, we report here a structural study of the dependence of the lattice parameters through the $\mathrm{RP} \rightarrow \mathrm{OG}$ transition region which, together with new specific heat data ${ }^{9}$ will serve to delineate more accurately the characteristics of this transition.

\section{EXPERIMENTAL AND DATA ANALYSIS PROCEDURES}

\section{A. Experiments}

The neutron-diffraction results reported here correspond to measurements carried out on the D4B two-axis diffractometer which was operated using two different incident wavelengths $\lambda_{0}$ of 0.705 and $0.499 \AA$ and on the D2B highresolution multidetector diffractometer with $\lambda_{0}=2.398 \AA$, at the Institut Laue Langevin, Grenoble. The different sets of measurements were needed to cover a large extent of momentum transfers, thus providing an acceptable resolution in the real-space quantities such as the static pair correlation functions. The maximum momenta transferred were 17, 24 (D4B), and $5 \AA^{-1}$ (D2B), respectively. The measurement on D2B was specially designed to improve the angular resolution ${ }^{10}$ in order to make the phases showing Bragg components amenable to Rietveld analysis.

On both instruments, the sample was held within a thinwalled vanadium container of $5 \mathrm{~mm}$ internal diameter and consisted of $99.8 \%$ deuterium-enriched anhydrous ethyl alcohol. Preliminary measurements on D4B which served to design the whole experimental procedure were also successfully completed employing a fully hydrogenous sample. In fact, in spite of the strong incoherent background, monitoring of the formation of the rotator-phase crystal could be conveniently followed even if the signal to background ratio was modest. The results were processed in the usual way and included corrections for container scattering, selfattenuation, and multiple scattering, achieving an absolute normalization of the cross section by means of comparison with the intensity scattered by a vanadium bar. The normalized cross sections for the liquid, glass and disordered crystal phases, all show a droop at large momentum ransfers $\left(Q \geqslant 6 \AA^{-1}\right)$ indicating a departure from the static approximation (inelasticity effects). The fact that such a decrease is found even at low temperatures $(5 \mathrm{~K})$ suggests that recoil processes involving molecular segments only are excited by the neutron beam (whole molecule recoil seems difficult to envisage in a solid at such low temperatures). Such effects need to be taken into account in order to remove the "selfcontribution" to the cross section (that not leading to interference effects which contains incoherent and coherent components) previous to transformation into real space quantities. To proceed, we have followed the procedure often employed for the analysis of molecular materials, ${ }^{11,7}$ which involves a parametric fit of a polynomial in even powers of $Q$ to the large- $Q$ portion of the structure factor. As an example, this correction for the low-temperature, normal liquid data described in this work can be accounted by a form $P(Q)=1-1.64 \times 10^{-3} Q^{2}+1.07 \times 10^{-6} Q^{4}$, so that the resulting estimate for the self-contribution will be given by $\left(6 \sigma_{\text {inc }}^{D} / 4 \pi+6 \overrightarrow{b_{D}^{2}}+2 \vec{b}_{C}^{2}+\vec{b}_{O}^{2}\right) \times P(Q)$, where $\sigma_{\text {inc }}$ stands for the nuclear incoherent cross section of the $D$ atoms and the $\overline{b_{i}}$ for the coherent scattering lengths of the $i$ th element.

All the phases were produced in situ using a standard ILL orange cryostat. Preparation of the amorphous phase was accomplished by simply plunging the cryostick (supporting the sample container) into the already-cold cryostat and then cooling the sample as quickly as possible, by which we achieved a cooling rate of 6 to $10 \mathrm{~K} / \mathrm{min}$. The plastic crystal or rotator-phase (RP) was then produced by annealing the glass or more precisely the supercooled liquid at about $108 \mathrm{~K}$ ( $T_{g} \approx 97 \mathrm{~K}$ ), which was then cooled below $97 \mathrm{~K}$ to produce the orientational glass (OG). Formation of the brittle (stable, monoclinic) crystal phase followed the melting of the RP crystal at $T \sim 140 \mathrm{~K}$ (where $T_{m}^{\mathrm{RP}} \approx 127 \mathrm{~K}$ ), and the stable crystal was finally melted to produce the liquid at even higher temperature $\left(T_{m} \approx 159 \mathrm{~K}\right)$.

The growth of rotator-phase crystals out of the supercooled liquid was monitored following the appearance of a sharp Bragg reflection at $Q \approx 1.675 \AA^{-1}$ which basically coincides with the maximum of $S(Q)$ for the supercooled liquid, which is a region of momentum-transfers free of reflections from the stable (monoclinic) crystal. ${ }^{4}$

The x-ray measurements were carried out using a Stoe powder diffractometer equipped with a continuous-flow nitrogen cryostat, and the sample was held in a thin-walled glass capillary attached to a goniometer head which was kept rotating around its vertical axis. The purpose of such measurements was to explore in detail the changes in the lattice parameters across the $\mathrm{RP} \rightarrow \mathrm{OG}$ transition. ${ }^{9}$ As shown in Ref. 1 the x-ray diffraction patterns of the disordered crystal phases exhibit only two reflections which index as (110) and (200) of a bcc lattice, whereas the neutron patterns show at least five clear Bragg peaks ${ }^{4}$ which index as (110), (200), (211), (220), (310), and a rather weak sixth (222) reflection which reveals itself only after a profile analysis. The positions of the first two peaks as well as their relative intensities are very similar in the neutron and $\mathrm{x}$-ray data, which can be taken as evidence of the large amount of orientational (rota- 
tional) disorder present in these two phases, otherwise far larger differences would be expected between the neutron and $\mathrm{x}$-ray spectra.

\section{B. Structure factors}

The single-differential radiation scattering cross section for a macroscopically isotropic system can be considered as a sum of intensities arising from different sources which contribute to the observed diffraction pattern with either sharp (Bragg) peaks arising from particles occupying, on average, well-defined equilibrium positions in a regular lattice (if it exists), a diffuse halo generated by disorder of dynamic (thermal motion), and/or static nature in cases where longrange regularities are absent, or a combination of both. The latter applies to systems where the presence of an underlying crystal lattice is concomitant with strong orientation disorder of either quenched (static disorder of molecular orientations) or dynamic (molecules sitting at the lattice nodes executing large amplitude rotations) origin. The $S(Q)$ static structure factors [obtained after correction of effects discussed in the previous section by normalizing the cross section by $N_{m}\left(\Sigma_{i} b_{i}\right)^{2}$, where $b_{i}$ stand for the nuclear coherent scattering lengths] are thus represented up to a reasonably good level of accuracy by a sum of plane (Bragg) and sphericalwave (diffuse) components, ${ }^{12-15}$

$$
S(Q)=S_{\text {Bragg }}(Q)+S_{\text {diff }}^{u}(Q)+S_{\text {diff }}^{c}(Q) .
$$

The $S_{\text {Bragg }}(Q)$ contains information about the time-averaged crystal structure, $S_{\text {diff }}(Q)$ does it for the deviation from such average structure, and is split into $S_{\text {diff }}^{u}(Q)$ giving the total diffuse intensity in the limit of no correlations between the constituent particles plus a modulation $S_{\text {diff }}^{c}(Q)$ comprising all those effects arising from the intermolecular interactions. The former quantity can be identified with ${ }^{15}$

$$
S_{\text {diff }}^{u}(Q)=f_{1}(Q)-f_{2}(Q) \exp (-2 W) .
$$

Here, $f_{1}(Q)$ is easily identified with the usual molecularform factor used in diffraction studies of liquids and is defined in terms of intramolecular parameters, ${ }^{11}$ so that the explicit form for $f_{1}(Q)$ is given by a sum of $j_{0}()$ 's weighted by the relevant concentration and scattering-length coefficients as well as by the appropriate intramolecular DebyeWaller terms defined in terms of the $\left\langle u_{i j}^{2}\right\rangle$ mean square amplitudes of thermal vibrations projected onto the vector joining nuclei $l$ and $m$, that is

$$
f_{1}(Q)=\frac{\sum_{l m} b_{l} b_{m} j_{0}\left(Q r_{l m}\right) \exp \left(-\left\langle u_{l m}^{2}\right\rangle Q^{2} / 2\right)}{\left(\Sigma_{l} b_{l}\right)^{2}} .
$$

Explicit formulas for the $f_{2}(Q)$ factor which involves correlations between different molecules can only be given in closed form for rather simple cases, such those where molecular orientations are assumed to be either totally uncorrelated or with very strong correlations. In the former case the total diffuse intensity would be calculable from knowledge of $f_{1}(Q)$ and the structure factor for the molecular centers of mass, ${ }^{14}$ whereas for the more likely case of orientations being correlated although statistically independent of their relative separation, such a term would involve averages over vectors specifying the relative position of nuclei belonging to different molecules which now become explicitly angle dependent.

A direct evaluation of the terms entering Eq. (1) thus seems quite a formidable task because of the limited amount of information present in the measured $S(Q)$ as well as the need of separating $S_{\text {diff }}^{u}(Q)$ from $S_{\text {diff }}^{c}(Q)$. Our approach instead proceeds in a stepwise manner, separating first the Bragg profile from the total $S(Q)$ by means of Rietveld analysis. The analysis of $S_{\text {Bragg }}(Q)$ then proceeds along established routes ${ }^{13,15}$ and provides information about the underlying crystal lattice. The difference $S(Q)-S_{\text {Bragg }}(Q)$ gives

$$
f_{1}(Q)+f_{2}(Q) \exp (-2 W)+S_{\text {diff }}^{c}(Q)
$$

from where subtraction of a $f_{1}(Q)$ form factor will provide the function $S_{\text {diff }}^{c}(Q)$ plus a term $f_{2}(Q) \exp (-2 W)$ which as shown by Gerlach and Prandl ${ }^{15}$ will contain contributions arising from the uncorrelated vibrations (translational) of the average molecule. Because of the Debye-Waller factor which gives mean-square amplitudes of about $0.17 \AA^{2}$, the latter function will decay quite fast having attained rather small values above $Q \geqslant 4-5 \AA^{-1}$.

As explained in detail in Ref. 16, because of cancellation effects it is expected that $S_{\text {diff }}^{c}(Q)$ will oscillate about zero and decay quickly (below $5 \AA^{-1}$ or so), but still giving rise to relatively well defined features in real space.

\section{Static correlations}

The expressions written above for the total structure factors can also be described in terms of reduced intensity functions, ${ }^{11}$ where contributions arising from incoherent and coherent contributions not giving rise to interference effects are subtracted from the total cross section

$$
Q I(Q)=Q[S(Q)-S(\infty)]=\frac{Q}{\left(\sum_{i} b_{i}\right)^{2}}\left(\frac{d \sigma}{d \Omega}\right)_{\text {interf }} .
$$

Similar equations can be written for the three different terms in Eq. (1) all of them having a correlate in real space since the Fourier sine transforms of such functions will give quantities such as

$$
\begin{aligned}
D(r) & =4 \pi \rho r[g(r)-1] \\
& =\frac{2}{\pi} \int_{0}^{Q_{\max }} d Q Q I(Q) \sin (Q r) M(Q),
\end{aligned}
$$

where $\rho$ stands for the atomic (or molecular) number density and $g(r)=\Sigma_{\alpha \beta} c_{\alpha} c_{\beta} b_{\alpha} b_{\beta} g_{\alpha \beta}(r)$ is the total radial distribution function comprised of partial correlations $g_{\alpha \beta}(r)$ between atoms $\alpha-\beta$ with relative concentrations $c_{\alpha, \beta}$ and scattering lengths $b_{\alpha, \beta}$, and $M(Q)=j_{0}\left(Q \pi / Q_{\max }\right)$ is a filter function employed to reduce the truncation error at $Q_{\max }$, that is the maximum momentum transfer allowed by the neutron kinematics. The sine-Fourier transform is then carried by Filon quadrature. Because of the oscillatory structure in the total $I(Q)$, some truncation error is inevitably expected to show up as spurious oscillations in the transformed $D(r)$ function. This being particularly noticeable for the total $D(r)$ 


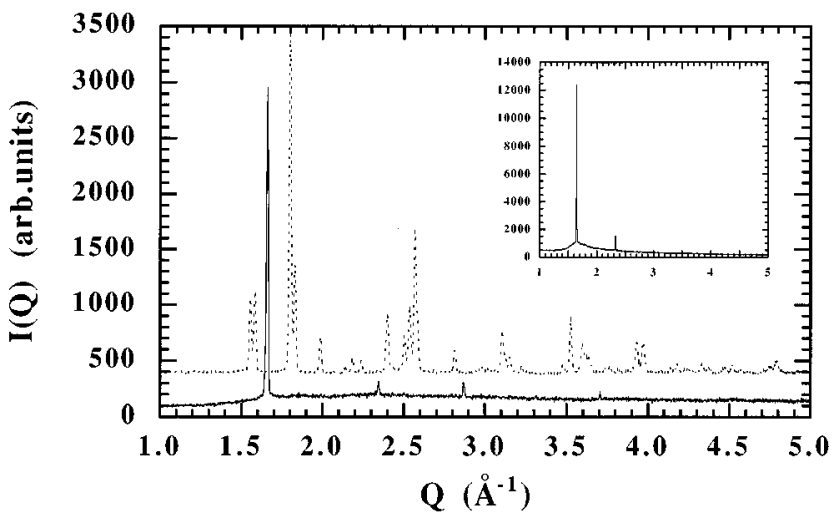

FIG. 1. The spectra of the monoclinic crystal (upper trace, vertically displaced) and plastic crystal (lower trace) measured under high angular resolution conditions on D2B. The inset shows the $\mathrm{x}$-ray pattern for the OG phase measured at $90 \mathrm{~K}$.

which shows some oscillations at $0.5 \AA$ or so, is substantially reduced in the intermolecular $D_{\text {inter }}(r)$ pair correlation function since subtraction of the molecular form-factor makes the integrand to die away at relatively low $Q$ 's as shown below. A comparison between the structure in real space of the total $D(r)$ and $D_{\text {inter }}(r)$ thus serves to ascertain the physical soundness of the main features. Finally, in order to check the correcteness of the normalization procedures the structure below $2 \AA$ was compared with the "density line" that is $-4 \pi \rho r$.

\section{RESULTS}

A comparison between the total $Q I(Q)$ functions for the stable crystal, glass, liquid, and the orientationally disordered crystal phases as measured on the D4B diffractometer using a wavelength of $\approx 0.7 \AA$ was shown in Fig. 1 of Ref. 4 . There it was shown that the most marked differences between glass and liquid spectra concern the position of the first peak $Q_{p}$, ascribable to a thermal expansion effect, leading to an expansion coefficient for the glass of $\approx 2.2 \times 10^{-4} \mathrm{~K}^{-1}$, comparable with those characteristic of known molecular glasses. ${ }^{19}$

Results measured under higher angular resolution with the D2B diffractometer for the monoclinic and RP crystals are now shown in Fig. 1. As can be seen, the rotator (and orientational glass) phases (a) show up to six Bragg peaks (the higher- $Q$ reflection is barely distinguishable from the diffuse background) and (b) that the peak width still is limited by resolution effects as a comparison with the $\mathrm{x}$-ray pattern given as inset exemplifies.

The $D(r)$ functions for the different condensed phases of this material are shown in Fig. 2. Inspection of such graphs shows that the functions for the glass, liquid and crystals are basically superimposable up to distances of $\approx 1.6 \AA$. This implies that, as can reasonably be expected, the characteristics of short-range order in glass and crystal are very close, or in other words, that crystal field effects are weak enough so that parameters regarding such distances should be transferable to the glass. On the other hand, the extent of the oscillations in real space for glass and liquid as compared with the disordered crystals become quite comparable to

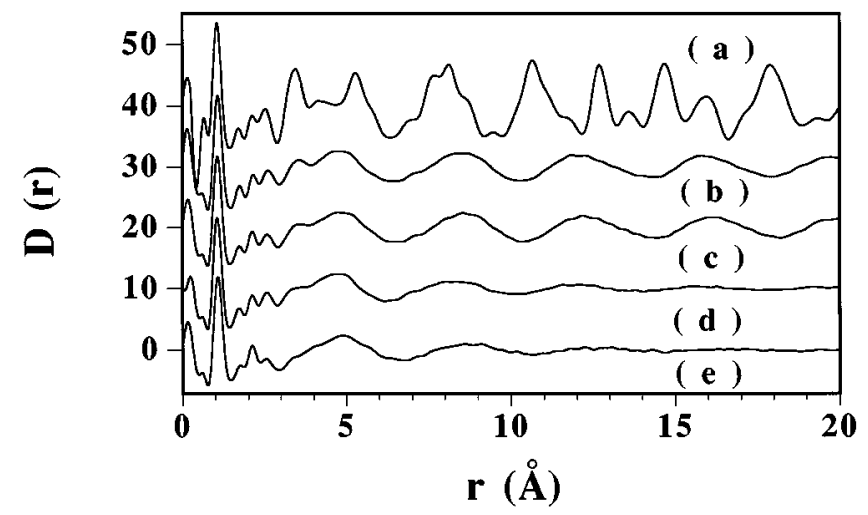

FIG. 2. A comparison of the total $D(r)$ static correlation functions corresponding to data measured on the $\mathrm{D} 4 \mathrm{~B}$ diffractometer using a wavelength of $0.5 \AA$. Units are atoms per $\AA^{-2}$. Curves a-d are upshifted by ten units.

those shown in Fig. 2 of Ref. 4 which were measured using a longer wavelength $(0.7 \AA)$ and therefore could have been affected by larger truncation errors.

In order to isolate the contribution $f_{2}(Q) \exp (-2 W)$ $+S_{\text {diff }}^{c}(Q)$ which accounts for the molecular correlations, the single-molecule $f_{1}(Q)$ form factor was subtracted from the measured interference functions. This was done following a procedure usually employed for the analysis of the structure factor of molecular materials, ${ }^{11}$ by means of a fit of a parametric form for $f_{1}(Q)$ to the large- $Q$ part [above $6 \AA^{-1}$ of $S(Q)$ (or $I(Q)]$.

The main thrust behind such effort was to explore the possibility of detecting some significant difference between the molecular structures in glass (or liquid) and crystal phases involving distances above $\approx 2.5-4 \AA$. Although, as referred above, large changes in bond-lengths and bond angles cannot sensibly be expected, the distribution of internal molecular configurations, that is those defined by the $C-C-O-D$ dihedral angle, surely departs form the 50-50 fraction of "trans" $\left(C-C-O-D \approx 180^{\circ}\right)$ and "gauche" $\left(C-C-O-D \approx 60^{\circ}\right)$ found in the crystal. This is a consequence of the relatively small difference in potential energy between the two configurations, which becomes comparable with that arising from the field produced by neighboring particles.

A comparison between experiment and model fits is shown in Fig. 3. A glance to the amount of information enclosed in the phase shifts present in the $Q I(Q)$ of Fig. 3 above $\approx 6 \AA^{-1}$, where the assumption that most of the scattering arises from within the molecular units holds, soon reveals that the number of $r_{i j}$ and $\left\langle u_{i j}^{2}\right\rangle$ parameters to be determined from the experimental intensities becomes too large. To alleviate this, we have set to the equilibrium crystal values the intramolecular bond lengths and angles as well as those regarding the $B$ thermal parameters ${ }^{6}$ upon scaling for mass and temperature differences. We have employed a common value for the $C-D$ bond length which was taken as an average over the crystal values, and the local symmetries of the $C D_{3}$ and $C D_{2}$ groups were assumed to belong to either $C_{3 v}$ or $C_{2 v}$ point groups. The values for the bond angles $C-C-O$ and $C-O-D$ where set to those given for the two crystal conformers. ${ }^{6}$ The main geometric parameter to be 


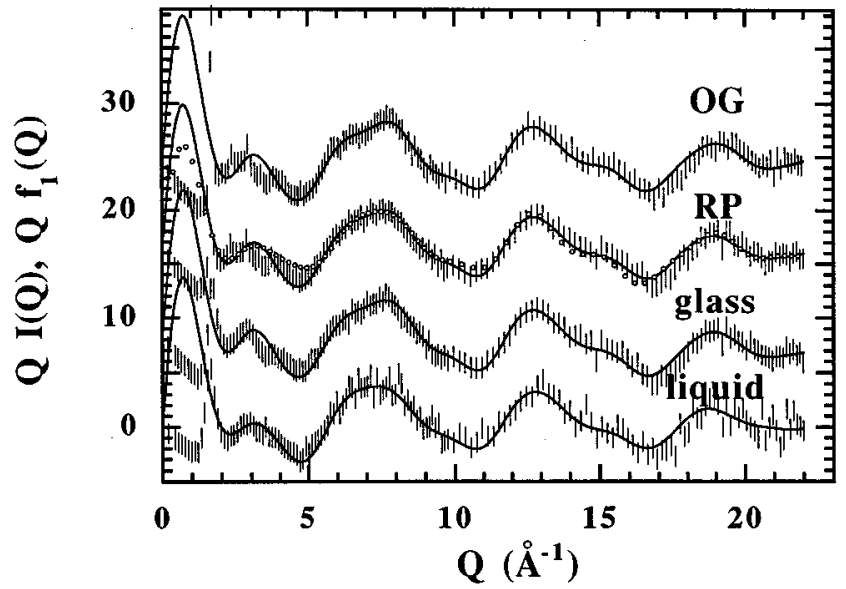

FIG. 3. An illustrative example of the approximation of the large- $Q$ region of the $Q I(Q)$ interference functions of the disordered solids and liquid by the $Q f_{1}(Q)$ molecular form-factors (full lines). The open symbols in the RP data correspond to the best estimate obtained using a single molecular ("trans") species only, and optimizing the rest of parameters in the same way as above.

optimized was therefore the $p$ which is the fraction of "trans" / "gauche" configurations alongside with variations of $\left\langle u_{i j}^{2}\right\rangle$ for atomic contacts corresponding to atoms separated by more than one bond.

The resulting fits of $Q I(Q)$ for all disordered phases shown in Fig. 3. The example given for the RP phase reveals that the experimental patterns are far better reproduced assuming the presence of two kinds of molecular conformers, specially at large $Q$ 's where the mismatch between experiment and model becomes specially severe (ie minimum at about $16.5 \AA^{-1}$ ). This confirms the presence within all the disordered phases of more than one molecular configuration, displaying a higher fraction of the "gauche" one, even if the proportion cannot be given accurately. This agrees with results of $\mathrm{Kakar}^{18}$ from microwave measurements, which shows a low "gauche-trans" energy difference about 5.1 $\mathrm{meV}$. At low temperatures we can expect that molecules choose a more compact conformation such as the "gauche" one. The fraction of the "trans" configuration was found to be 0.25(12), 0.26(23), and 0.24(19) for the RP liquid and glass, respectively. This contrasts with previous neutron and x-ray diffraction studies within the normal liquid, ${ }^{7}$ where the presence of only one configuration was postulated, a result which can also be understood by examination of data shown in Fig. 3, because of the more smeared out structure of the liquid $Q I(Q)$.

The patterns for the orientationally ordered crystal newly measured on the D2B were analyzed by means of a Rietveld profile refinement. ${ }^{17}$ The geometry and thermal parameters given by Jönsson ${ }^{6}$ were used, with the only exception of the thermal vibration amplitudes entering the Debye-Waller terms which were rescaled to the temperature under consideration $(105 \mathrm{~K})$ as well as by the isotopic mass difference between deuterated and hydrogenated samples. The measured profile could be reproduced quite satisfactorily, although some differences in the peak amplitudes of largeangle reflections between the model and experiment, probably due to the presence of significant texture, could not be reconciled. Therefore, only data regarding thermal expansion effects have been derived for this phase. The structure at both low and high temperatures conforms to a monoclinic $P c$ space group with four molecules per unit cell with parameters $a=5.281 \AA, \quad b=6.755 \AA, \quad c=8.180 \AA \quad$ and $\beta=102.06^{\circ}$ at $T=10 \mathrm{~K}$ which gives a volume of $285.4 \AA^{3}$, whereas the results for $140 \mathrm{~K}$ are $a=5.322 \AA, b=6.911 \AA$, $c=8.245 \AA, \beta=101.04^{\circ}, V=303.2 \AA^{3}$. This shows a volumic contraction of $6.2 \%$ upon decreasing the temperature by $130 \mathrm{~K}$, that is from about $19 \mathrm{~K}$ below melting. The results for $105 \mathrm{~K}$ on $\mathrm{D} 2 \mathrm{~B}$ are $a=5.350 \AA, b=6.859 \AA$, $c=8.232 \AA, \beta=101.42^{\circ}$ which give a volume of $296.1 \AA^{3}$, and therefore represent a contraction of about $2.4 \%$. This provides an indication of the strong anharmonic regime governing the molecular interactions even in the crystalline "ground state" at temperatures where both the supercooledliquid to glass and the $\mathrm{RP} \rightarrow \mathrm{OG}$ transitions take place.

\section{A. Orientational distributions and molecular correlations in the rotator and orientational-glass phases}

High-resolution measurements for the orientationally disordered crystals were carried on D2B for a set of temperatures of $95,100,105$, and $110 \mathrm{~K}$ to provide information more detailed than that already available for $S_{\text {Bragg }}(Q)$, which was ostensibly resolution limited. This was needed for establishing with greater detail the time-averaged distribution of molecular orientations as well as to put some bounds to the quantity $S_{\text {diff }}^{c}$, comprising correlations between different molecules.

From the measured Bragg patterns values for the lattice parameter for the bcc structure are derived ${ }^{20}$ and go from $5.333 \AA$ at the lower temperature to $5.354 \AA$ at the highest. This gives an expansion coefficient of about $2.6 \times 10^{-4} \mathrm{~K}^{-1}$, which is remarkably close to that of the glass. It also seems worth remarking the close similarity of the structure factors at both sides of the rotational freezing transition, where most differences are found in the contribution of diffuse origin leaving the pattern of Bragg reflections barely affected. This is easily understandable if results reported in Ref. 3 regarding the dynamic structure factors of both RP and OG are taken into account. There, it is seen that the main difference in the dynamic response of both phases concerns the presence in the rotator phase of a broad, wave-vector independent, quasielastic component arising from molecular rotations, which will only contribute to the diffuse intensity.

To proceed, the pattern of Bragg reflections is first isolated from the total profile by means of Rietveld ${ }^{17}$ analysis and subsequently, the Bragg pattern is analyzed in terms of several single-molecule models. Such level of description is justified by the rather different site and molecular symmetries which force the molecule to adopt a number of equilibrium orientations in order to satisfy the site symmetry, and such orientations are attained in the rotator phase by means of motions around an average orientation. For the RP this approach will provide an approximation of the motion of a molecule in its surroundings, whereas for the $\mathrm{OG}$ it will give information about the extent of the quenched orientational disorder within the bcc lattice. In both cases, one molecule must lie at the cell origin and the other at the center of the cell as schematically shown in Fig. 4. This requires the crys- 


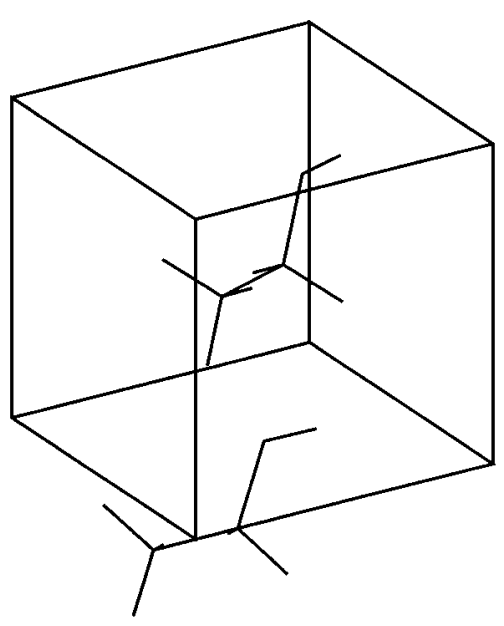

FIG. 4. A snapshot of the bcc cubic unit cell of ethanol (for the OG and RP phases), which shows only the two independent molecules which define the unit cell.

tal space group to be symmorphic, i.e., the crystal symmetry operations must be purely rotational (modulus a Bravais lattice translation) so that the molecular position at the cell origin is invariant under all symmetry operations. We find for ethanol five bcc space groups which fulfil these conditions, namely, $I 23, \operatorname{Im} \overline{3}(m \overline{3}$ Laue class) and $I 432, I \overline{43} m$, $\operatorname{Im} \overline{3 m}(\mathrm{~m} \overline{3 m}$ Laue class). Between those, as experience dictates $^{21-23}$ the space group most likely to provide a faithful representation of the OG and RP structures is the one with the maximum allowed symmetry within the Laue class. This property stems from the high entropy arising from dynamical disorder characteristic of plastic (rotator) crystals, and hence the space group must generate a large number of symmetryequivalent molecular orientations. In our case, the highestsymmetry space group would be $\operatorname{Im} \overline{3 m}$. Most rotator-phase crystals have the striking property that the molecular site symmetry is higher than the molecular point-group symmetry itself. ${ }^{24}$ This is a direct consequence of the dynamical disorder, i.e., the molecule performs successive reorientations between symmetry related positions so that the timeaveraged structure conforms to the crystal site symmetry. In our case, the site symmetry would be $m \overline{3} m\left(\mathcal{O}_{h}\right)$ whereas the molecular point symmetry $\left(\mathcal{C}_{1 h}\right)$ has, at most, a mirror plane element for the molecular trans conformation.

The diffraction profile was fitted to a set of $(k h l)$ intensities, cell parameter, background, and peak-shape (instrumental) functions assuming the $m \overline{3} m$ Laue class. The background is reproducible using a cubic polynomial and a Lorentzian peak shape accounts for the peak shape and gives a fitted full width at half maximum value of $0.29^{\circ}$. The refined value of the cubic lattice parameter is 5.369(1) $\AA$ with a weighted disagreement factor $R_{w}=3.6 \%$. The values obtained for the six observed Bragg intensities are displayed in Table I together with the observed values and the computed deviations.

The simplest approach which is commensurate with the site symmetry corresponds to an isotropic (continuous) distribution of molecular orientations, which as already shown ${ }^{4}$ takes account of the oscillations appearing in the experimental $D(r)$ from about $3.7 \AA$ onwards. The origin of the peak
TABLE I. Experimental Bragg intensities of the cubic phase of ethanol at $90 \mathrm{~K}$ extracted from the powder diffraction spectra with a profile matching procedure. The three rightmost columns show a comparison of the Bragg intensities as calculated from an isotropic model with the experimental pattern expressed in absolute units, and the calculated standard deviation

\begin{tabular}{lrrrr}
\hline \hline$(h k l)$ & Intensity & \multicolumn{1}{c}{$F_{\text {calc }}$} & $F_{\text {obs }}$ & $\sigma$ \\
\hline$(110)$ & $1013(12)$ & 31.89709 & 31.83400 & 0.19469 \\
$(200)$ & $57(6)$ & 6.02398 & 7.53558 & 0.42909 \\
$(211)$ & $32(5)$ & 2.73132 & 5.64305 & 0.49193 \\
$(220)$ & $6(3)$ & 5.11197 & 4.48817 & 0.74070 \\
$(310)$ & $17(4)$ & 4.30463 & 4.16857 & 0.51588 \\
$(222)$ & $3(3)$ & 1.95492 & 1.89865 & 0.87632 \\
\hline \hline
\end{tabular}

about $3.5 \AA$ or so can be ascribed to molecular orientational correlations, as discussed below.

As far as how a single molecule explores all available orientations, two alternatives can be tested. The simplest assumes isotropic rotational diffusion so that the atoms distribute uniformly in concentric spherical shells. Alternatively a discrete representation of the available sites from within which very fast jumps between symmetry-related molecular orientations take place can also be used. The latter is usually referred to as the Frenkel model.

To model the RP crystal we have first considered an isotropic spherical orientational distribution where the center of the sphere is found by a least-squares refinement of the observed Bragg intensities. An anisotropic thermal parameter (Debye-Waller factor) was also introduced but the refinement process became unstable and a fixed reasonable value of $\left\langle u_{x}^{2}\right\rangle=0.019 \AA^{2}$ was chosen in further refinements. The scarce number of Bragg reflections in our case makes the fitting procedure a complicated task as local minima abound. The refinements converge to a solution which fits well the six Bragg intensities with a weighted factor of $R_{w}=2.2 \%$. The spherical rotation center is finally found to be close to the molecular center, thus leading to a minimum effective volume. Such a result is also supported by the presence within the RP phase of a basically $Q$-independent quasielastic background, as well as with the similarity of the x-ray and neutron-diffraction intensities. This suggests that the contribution of the deuterium atoms is very small, as it happens here, if they distribute uniformly in the outer spherical shells. If they were localized their contribution would be even larger than that of the skeletal atoms as it happens for the monoclinic phase. Also, this result seems to confirm that the correct space group is $\operatorname{Im} \overline{3 m}$ as a spherical distribution shows the maximum allowed symmetry.

Attempts have also been made to fit a Frenkel model assuming the $\operatorname{Im} \overline{3 m}$ space group. Here, a given molecular position generates 48 equivalent orientations. Because of this large number, the atoms spread rather densely over spherical shells during their motion and in practice this model approaches the previous one. A systematic search of the parameter space (molecular orientation and translation) has been performed in order to localize the least-squares minimum with respect to the observed intensities but many different equally good solutions were found. This multiplicity is surely due to the aforementioned fact that all of them result 

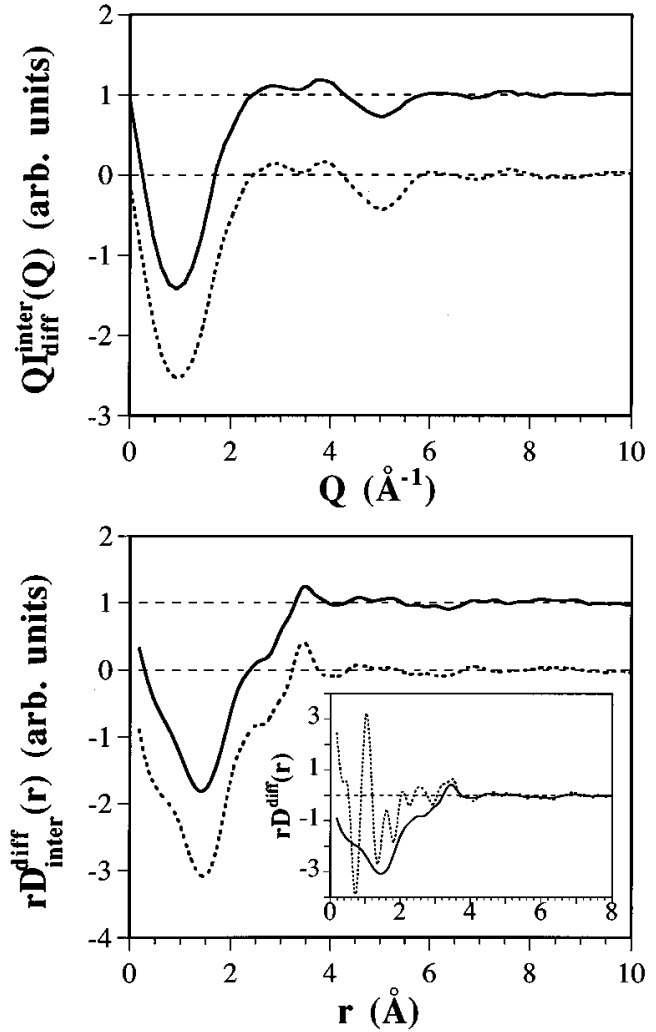

FIG. 5. The upper frame shown the $Q I_{\text {diff }}^{\text {inter }}(Q)$ comprising the center of mass and orientational correlations. The upper frame shows the diffuse scattering intensities for the RP (solid line) and the OG (dashed line) arising from molecular correlations as given by Eq. (4). The upper curve has been upshifted by one unit to facilitate viewing. The lower frame displays the $D_{\text {inter }}^{\text {diff }}(r)$ real space correlates of the functions shown in the upper frame. The inset depicts a comparison between one of the latter functions and the one arising from Fourier-inverting the total diffuse intensity (dots).

in an almost uniform orientational distribution. The disagreement factor is very low $(\sim 0.8 \%)$ and the calculated intensities are within the experimental uncertainties. In all cases the molecular center converges towards the cell origin thus minimizing the time-average molecular effective volume.

The next step of the analysis involves the establishment of the relative importance of the intermolecular correlations as represented by $S_{\text {diff }}^{c}(Q)$. Since, as described above, the single-molecule contribution $S_{\text {diff }}^{u}(Q)$ to the diffuse intensity has already been taken care of, here we are set to isolate the rest of contributions entering Eq. (4) by experimental means. This can be achieved after subtraction of $f_{1}(Q)$ as well as the Bragg profile to the experimental spectra. What is left is a function

$$
I_{\text {diff }}^{\text {inter }}(Q)=S_{\text {diff }}^{c}(Q)+f_{2}(Q) \exp (-2 W)-S(\infty)
$$

which shows oscillations up to about $10 \AA^{-1}$ and an example of those for the RP and OG crystals is given in Fig. 5. Such functions once transformed into real space give rise to a function $D_{\text {inter }}^{\text {diff }}(r)$ which basically has only two welldefined features, being a shoulder at about $2.5 \AA$ [a distance reminiscent of nearest-neighbor (hydrogen-bonded) contacts in the monoclinic crystal], and a rather well-defined peak at

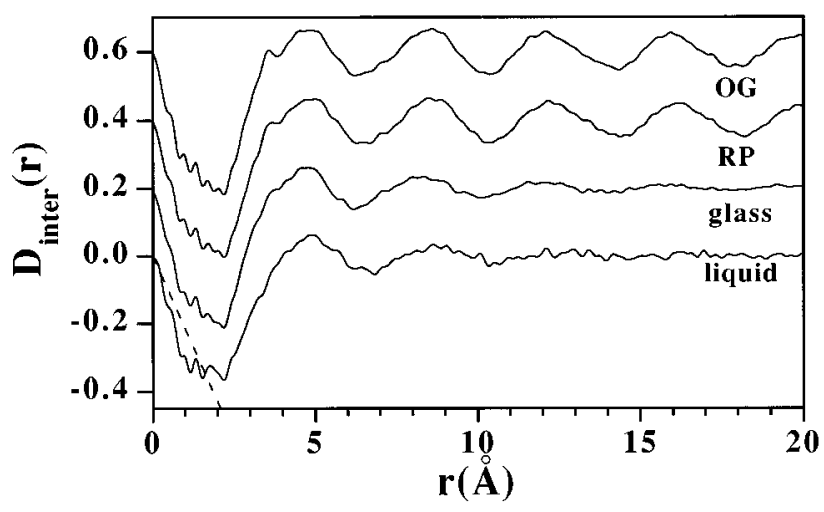

FIG. 6. Static $D_{\text {inter }}(r)$ intermolecular pair correlation functions for the orientational glass, rotator phase crystal, glass, and normal liquid. The dashes represent the density line $-4 \pi \rho r$ for the liquid which is given in molecules per $\AA^{-2}$. All other curves have been upshifted by 0.2 units increments.

$\approx 3.5$ A. A glance at Fig. 2 shows that the latter peak has an intensity which seems to be quite directly related to the extent of orientational randomness, being minimal for the normal liquid and maximal for the stable crystal. It is the only intermolecular feature which is narrow and intense, different from those produced by long-range regularities. Its assignment to specific atomic contacts was attempted on the basis of some reverse Monte Carlo calculations and found to arise from a mixture of the partials $g_{D D}(r), g_{C D}(r), g_{O O}(r)$, and $g_{O D}(r)$, none of them being dominant. The importance of $D_{\text {inter }}^{\text {diff }}(r)$ can be quantified with respect to the transform of $D_{\text {tot }}^{\text {diff }}=(2 / \pi) \int d Q Q\left[I(Q)-I_{\text {Bragg }}(Q)\right] \sin (Q r)$ which gives all the contributions arising from the diffuse intensity. Such a comparison is also provided as an inset to Fig. 5 and shows that $D_{\text {inter }}^{\text {diff }}(r)$ dominates above $3.2 \AA$ or so, even if the ratio of the integral of that function with respect to that arising from the total diffuse intensity amounts a meagre $7.5 \%$ only in the RP and about $8.4 \%$ in the frozen OG phase.

\section{B. Intermolecular structure for the liquid and glass in relation to that of the RP and OG phases}

The static $D_{\text {inter }}(r)=4 \pi \rho r\left[g_{\text {inter }}(r)-1\right]$ pair correlation functions for intermolecular contacts for all the disordered phases are shown in Fig. 6. These are the Fourier pairs of $Q\left[S(Q)-f_{1}(Q)-S(\infty)\right]$ and represent the extent of correlations of both positional and orientational nature. As far as the former are concerned, the low-period oscillation with first maxima appearing at about $4.5 \AA$ in the $\mathrm{OG}$ and $\mathrm{RP}$ functions constitute the more clear signature. They originate from the set of Bragg peaks as discussed in more detail in Ref. 4. These peaks appear because of an underlying timeaveraged crystal structure, which exists even if at some given instant deviations from it can be relatively large due to the large amplitude motions executed by individual molecules in some of the disordered phases (RP). Such timeaverage structure is not very much different from the ensemble-averaged cubic crystal structure characteristic of the OG phase, where only small amplitude librations take place. Taking such an analogy a step further one can relate the low-period oscillations in the glass and liquid $D_{\text {inter }}(r)$ 
appearing as maxima at about $\approx 4.5$ and $\approx 9 \AA$ to the presence of the first, strongest peak in $S(Q)$, and theretofore to the existence of a time and ensemble-averaged intermediaterange regularity which only concerns the molecular centers of mass. Such regularities are, on the other hand relatively modest in their extent, and never surpass characteristic scales of about $20 \AA$, whereas those for the RP and OG phases should extend over several hundreds of ångströms, a guess made from the estimated natural widths of the Bragg peaks.

In parallel, the decrease in orientational correlations in passing from the disordered crystals to the glass and liquid can also be followed from Fig. 6, together with information about $S_{\text {diff }}^{c}(Q)$ discussed in the previous section. The referred figure shows that the characteristic peak appearing at about $3.5 \AA$ in the disordered crystals now appears as a shoulder in the glass function and is basically blurred in the normal liquid, where it would become rather hard to quantify.

The present result thus shows how correlations of both positional and orientational origin are enhanced when crossing both the canonical liquid $\rightarrow$ glass transition alongside with the $\mathrm{RP} \rightarrow \mathrm{OG}$ freezing, and therefore those of purely orientational nature will have to be considered on the same footing as those of positional origin.

\section{Rotational freezing transition}

To investigate in more detail the structural changes associated with the $\mathrm{RP} \rightarrow \mathrm{OG}$ transition a series of $\mathrm{x}$-ray diffraction measurements at temperatures comprising the transition region were carried out. From there the temperature dependence of the $d$ spacing corresponding to the most intense (110) diffraction peak was derived after profile analysis of the diffraction patterns. The results are shown in Fig. 7 together with calorimetric data ${ }^{9}$ which show the variation of the heat capacity for the monoclinic crystal in a range of temperatures where the $\mathrm{RP} \rightarrow \mathrm{OG}$ transition as well as the canonical liquid $\rightarrow$ glass takes place. As can be seen from comparison of the $d(T)$ and $C_{p}(T)$ graphs for the $\mathrm{RP} \rightarrow \mathrm{OG}$ transition, the latter appears somewhat more smeared out if followed through the structural parameter than that witnessed by the associated changes in the heat capacity, where the jump in $C_{p}$ is confined to a region of about $9 \mathrm{~K}$, quite comparable in fact to the range covered by the conventional glass transition. Such smearing out of the transition surely arises from both the strong thermal expansion effects referred in previous sections as well as from the plausible gradual freezing of the rotational degrees of freedom.

In this latter respect, one sees that at the lower end of the transition, $90 \leqslant T \leqslant 95 \mathrm{~K}$, the heat capacity of the disordered crystal significantly exceeds that of the fully disordered material, something which provides a clear indication of the sluggish arrest of the orientational degrees of freedom. In fact, the specific heat of the OG phase remains slightly above that of the glass down to about $10 \mathrm{~K}$, a fact explainable by the somewhat different shapes of the generalized frequency distributions. ${ }^{3}$ Notice however that such an excess in heat capacity does not imply an entropy excess of the disordered crystal over the glass, thus leading to a Kauzmann paradox. In fact ${ }^{2}$ the entropy of the OG crystal is bounded below that of the glass and supercooled liquid over the entire temperature range. A point which seems worth commenting in this

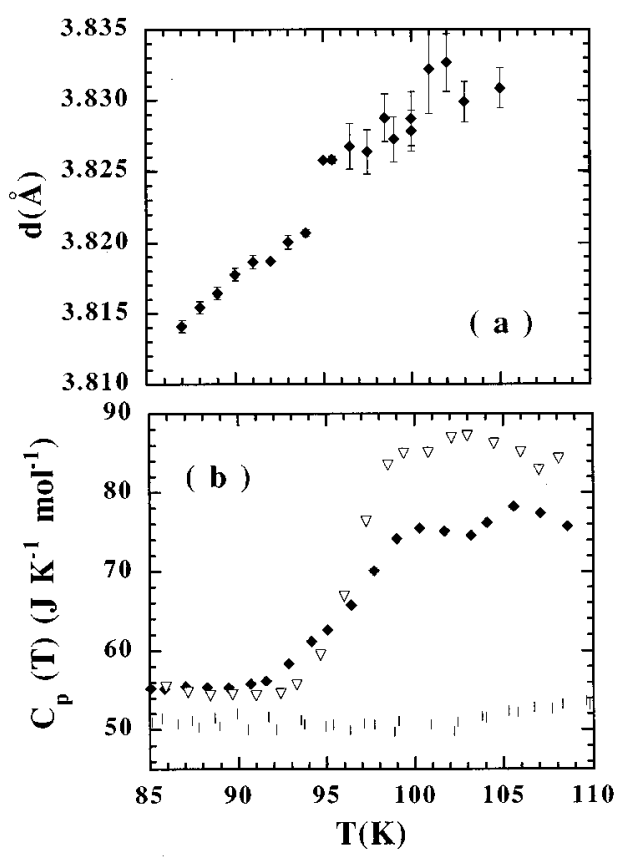

FIG. 7. (a) The variation of the lattice spacing corresponding to the main peak of $S(Q)$ along the $\mathrm{RP} \rightarrow \mathrm{OG}$ transition region. (b) Accompanying changes in the constant-pressure heat capacity Ref. 9 for the stable (monoclinic) crystal (vertical bars) $\mathrm{RP} \rightarrow \mathrm{OG}$ transition (filled symbols) as well as the supercooled liquid $\rightarrow$ glass (open symbols).

respect is the value of the entropy change at the transition, which gives $\Delta S=12.84 \mathrm{~J} \mathrm{~K}^{-1} \mathrm{~mol}^{-1}$ or $\approx 3 \ln 2$ per formula unit, as expected for a purely rotational melting process.

The apparent discontinuity at about $95 \mathrm{~K}$ exhibited by $d(T)$ could not be fully ascertained because the relatively poor statistics of data above such temperatures, possibly due to some small hysteresis effect.

\section{DISCUSSION AND CONCLUSIONS}

Monitoring the changes in orientational and positional correlations in passing from the fully ordered crystal to the glass has been made possible by the existence of the orientationally disordered phases of solid ethanol, which constitute states showing positional long-range order only. From data given in previous sections the following points are shown.

The realm of orientational correlations in the glass phase is confined to next-nearest neighbors, at most. This is so even for the disordered crystal (RP and OG) phases, where no signature of correlations of this kind appears for distances well above $8 \AA$ or so,

In contrast, positional correlations extend in the glass phase up to $15 \AA$ or so, and up to about $10 \AA$ in the lowtemperature, normal liquid. This has been ascertained by the close relationship between the low-period oscillations appearing in the $D(r)$ functions of the glass (and) liquid with those of the orientationally disordered phases, once the orientational correlations are accounted for.

In consequence, the presence of IRO is shown here to arise from mainly positional correlations, and this involves 
the glass neighboring particles located up to a third shell from the origin.

Correlations of orientational origin are, however, relatively more enhanced than the positional ones upon crossing from above the liquid-glass and orientational freezing transitions. The contribution of $D_{\text {inter }}^{\text {diff }}(r)$ to the total correlation function $D_{\mathrm{tot}}^{\text {diff }}(r)$ has served to quantitatively express this for the $\mathrm{RP} \rightarrow \mathrm{OG}$ transition. Such an enhancement can only be discussed on qualitative grounds, on the basis of the feature appearing at $3.5 \AA$, in the case of the canonical liquid-glass transition.

The implications of the present results should be clear for those materials composed of well-defined stoichiometrical units, irrespective of whether these are of molecular nature or not (e.g., $\mathrm{SiO}_{4}$ tetrahedra or $\mathrm{B}_{2} \mathrm{O}_{3}$ groups), and in fact, some analogies with the premelting behavior of narrow-gap semiconductors can be found. ${ }^{26}$ Also, the present results provide a basis for the understanding of some recent findings ${ }^{27}$ where the most noticeable structural change in a deeply supercooled liquid was found to be related to the increase of orientational correlations.

The $\mathrm{RP} \rightarrow \mathrm{OG}$ transition can be considered as a physical realization of the hard needles on a fcc lattice model explored by Renner et $a l .{ }^{28}$ which exhibits a kinetic glass transition when $l=L / a$, the needle length to lattice constant ratio, exceeds about 2.7. As should be evident, the control parameter in our case cannot be the same $l$, valid for a model of noninteracting bodies and which in our case is about 0.72 but rather the temperature, which below about $90 \mathrm{~K}$ leads to a complete arrest of the large amplitude rotational motions. A direct experimental test of that dynamic arrest was pro- vided by inelastic neutron-scattering results given in Ref. 3 where the broad quasielastic background of rotational origin was shown to remain well into the OG phase. Whether the sluggish thermally activated orientational librations referred to in Ref. 28 taking place close to criticality can be identified with some motions giving rise to an excess of $C_{p}$ in the temperature range of $86 \leqslant T \leqslant 95 \mathrm{~K}$ where, as referred above, $C_{p}^{O G}>C_{p}^{\text {glass }}$, constitutes an interesting topic of future research. Such behavior may also be related to the presence of sub- $T_{g}$ relaxations in analogy with those found in other rotator-phase compounds. ${ }^{25}$

As shown in previous sections, the freezing of the rotational degrees of freedom characteristic of the RP phase occurs over a range of temperatures remarkably similar to that comprising the canonical $T_{g}$ without any clearly measurable discontinuity in the cell volume. Other static properties examined so far also show a lack of any relevant discontinuity. Therefore, the freezing transition seems to have a dynamic origin and hence provides a very adequate testing ground for the predictions made from mode-coupling approaches. ${ }^{29,30}$

\section{ACKNOWLEDGMENTS}

This work was performed in part under DGICYT (Spain) Grant No. PB95-0072-C03-01. The authors would like to thank M. González, P. Radaelli, P. Cross, and P. Palleau of the Institut Laue Langevin for their scientific and technical assistance as regards the neutron diffraction experiments. M.A. Ramos and S. Vieira are acknowledged for the specific heat data.
${ }^{1}$ A. Srinivasan et al., Phys. Rev. B 53, 8172 (1996).

${ }^{2}$ O. Haida, H. Suga, S. Seki, J. Chem. Thermodyn. 9, 1133 (1977); M. Hugo, K. Schlüter, and A. Würflinger, Z. Phys. Chem. (Neue Folge) 175, 235 (1992).

${ }^{3}$ M. A. Ramos et al., Phys. Rev. Lett. 78, 82 (1997); in NonEquilibrium Phenomena in Supercooled Liquids, Glasses and Amorphous Materials (World Scientific, Singapore, in press).

${ }^{4}$ R. Fayos et al., Phys. Rev. Lett. 77, 3823 (1996).

${ }^{5}$ For recent appraisals, see, P. S. Salmon, Proc. R. Soc. London, Ser. A 445, 351 (1994); P. H. Gaskell and D. J. Wallis, Phys. Rev. Lett. 76, 66 (1996).

${ }^{6}$ P. G. Jönsson, Acta Crystallogr., Sect. B 32, 232 (1976).

${ }^{7}$ D. G. Montague, I. P. Gibson, and J. C. Dore, Mol. Phys. 47, 1405 (1982); Y. Tanaka, N. Ohtomo, and K. Arakawa, Bull. Chem. Soc. Jpn. 57, 2569 (1984); A. H. Narten and A. Habenschuss, J. Chem. Phys. 80, 3387 (1984).

${ }^{8}$ T. Eguchi et al., Mol. Phys. 40, 681 (1980).

${ }^{9}$ F. J. Bermejo et al., in Complex Behaviour of Glassy Systems, edited by M. Rubí et al., Springer Lecture Notes in Physics (Springer-Verlag, Berlin, in press). The heat capacity excess of the OG with respect to the glass within the range $80 \leqslant T \leqslant 95 \mathrm{~K}$ is also seen in data reported in Fig. 4 of H. Suga, Pure Appl. Chem. 55, 427 (1983).

${ }^{10}$ Institut Max von Laue Paul Langevin, Guide to Neutron Research Facilities at the ILL, edited by K. Ibel (ILL, Grenoble, France, 1994).
${ }^{11}$ J. G. Powles, Adv. Phys. 22, 1 (1973).

${ }^{12}$ M. A. Krivoglaz, X-Ray and Neutron Diffraction in Nonideal Crystals (Springer-Verlag, Berlin, 1996), p. 241.

${ }^{13}$ M. T. Dove and G. S Pawley, J. Phys. C 17, 6581 (1984).

${ }^{14}$ P. A. Egelstaff, D. I. Page, and J. G. Powles, Mol. Phys. 20, 881 (1971).

${ }^{15}$ P. Gerlach and W. Prandl, Acta Crystallogr., Sect. A 44, 128 (1988).

${ }^{16}$ F. Dunstetter, Ph.D. thesis, Universite de Paris-Sud, Orsay, 1988; F. Dunstetter and A. Delapalme, Physica B 156-157, 11 (1989); Fiz. Nizk. Temp. 22, 119 (1996).

${ }^{17}$ Program FULLPROF. A version of the Rietveld code developed by J.Rodríguez-Carvajal. Obtainable from Laboratoire Leon Brillouin, C.E.N. Saclay, 91191 Gif sur Yvette, France.

${ }^{18}$ R. K. Kakar and C. R. Quake, J. Chem. Phys. 72, 4300 (1980); G. Dolling, B. M. Powell, and V. F. Sears, Mol. Phys. 37, 1859 (1979).

${ }^{19}$ W. A. Phillips, in Amorphous Solids: Low-Temperature Properties, edited by W. A. Phillips (Springer-Verlag, Berlin, 1981), p. 53.

${ }^{20}$ Program LSUCREB (D.E. Appleman, U.S. Geological Survey, Washington D.C.).

${ }^{21}$ D. André, D. Ceccaldi, and H. Szwarc, J. Phys. (France) 45, 731 (1984).

${ }^{22}$ J. P. Amoureux, M. Beé, and J. L. Sauvajol, Acta Crystallogr., Sect. B 38, 1984 (1982). 
${ }^{23}$ R. Fourme, J. Phys. (France) 40, 57 (1979).

${ }^{24}$ The Plastically Crystalline State, edited by J. N. Sherwood (Wiley, Chichester, 1979).

${ }^{25}$ M. Descamps et al., in Quasielastic Neutron Scattering, edited by J. Colmenero et al. (World Scientific, Singapore, 1994), p. 107; Physica A 201, 346 (1993).

${ }^{26}$ D. L. Price, M. L. Saboungi, and W. S Howells, Phys. Rev. B 51, 14923 (1995).
${ }^{27}$ R. Leheny et al., J. Chem. Phys. 105, 7783 (1996).

${ }^{28}$ C. Renner, H. Löwen, and J. L. Barrat, Phys. Rev. E 52, 5091 (1995).

${ }^{29}$ E. R. Duering, R. Schilling, and H. P. Wittmann, Z. Phys. B 100, 400 (1996).

${ }^{30}$ W. Gotze, in Liquids, Freezing and the Glass Transition, edited by J. P. Hansen et al. (North-Holland, Amsterdam, 1991), p. 287. 